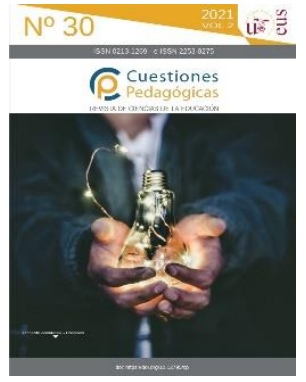

\title{
La defensa de los territorios como parte de la propuesta educativa del Instituto Superior Intercultural Ayuuk
}

\author{
Dispute for the territories of Indigenous Peoples and their \\ young people. Instituto Superior Intercultural Ayuuk
}

\author{
Alma Patricia Soto-Sánchez \\ CONACYT (México) \\ altzilal@gmail.com \\ https://orcid.org/0000-0003-2459-4731
}

Resumen: Los Pueblos indígenas en México enfrentan actualmente la intensificación de conflictos socioambientales frente a proyectos históricos de desarrollo y extractivos que generan despojo. En el presente artículo se analiza el Instituto Superior Intercultural Ayuuk (ISIA) formando parte de diversos procesos que convergen en el objetivo de favorecer la permanencia de las comunidades; se presenta su origen en procesos de organización social de defensa de los territorios, su propuesta de educación intercultural desde el ejercicio del derecho de los Pueblos a tener su propia educación, así como su área de influencia, que presenta conflictos y tensiones frente a la geopolítica del saber y el poder. Se reflexiona, desde este marco histórico y geopolítico, el Seminario de Titulación (SETI), de la Licenciatura en Administración y Desarrollo Sustentable, como espacio significativo de síntesis y creatividad, donde las y los estudiantes diseñan una metodología de investigación, acción participativa (IAP), creando comunidades de aprendizaje en sus comunidades de origen que generan procesos de reflexión que fortalecen 0 generan procesos de defensa y cuidado de sus territorios. La información presentada se recopiló a través de una investigación colaborativa con el ISIA, en el marco del proyecto "Políticas de Interculturalidad en
Abstract: Indigenous Peoples in Mexico are currently facing the intensification of socioenvironmental conflicts in the face of historical development and extractive projects that generate dispossession. In this article, the Ayuuk Intercultural Superior Institute (ISIA) is analyzed as part of diverse processes that seek as common goal the permanence of the communities. The paper presents ISIA's origin in asocial organization process for the defense of indigenous territories, their area of influence that presents conflicts and tensions in the face of the geopolitics of knowledge and power, and the importance of this proposal for intercultural education as the right of Indigenous People to decide their own education. It is particularly presented the Seminar of Qualification (SETI), of the Administration and Sustainable Development graduate program, as a relevant space for synthesis and creativity, where the students design participatory action research methodologies to create learning communities within their own communities, generating reflection processes that strengthen or generate new processes of defense and care of their territories. The information presented was compiled through a collaborative-research, within the project Interculturality Policies in higher education in Oaxaca: ethnopolitical educational projects", from CONACYT/ CIESAS Pacífico Sur 
educación superior en Oaxaca: proyectos

educativos etnopolíticos", del

CONACYT/CIESAS Pacífico Sur.

Palabras clave: Educación Superior Keywords: Intercultural Superior Education, Intercultural, pueblos Indígenas, jóvenes Indigenous People, indigenous territories and indígenas. youth, geopolitics, ethnopolitics.

\section{Introducción}

En años recientes, los conflictos socioambientales en los territorios de los Pueblos indígenas se han intensificado por procesos históricos que se recrudecen en esta nueva ola de expansión del capital, que genera la explotación de mano de obra y de la naturaleza; (Avila y Avila, 2017; Bartra, 2008; Barrón, 2010; Bonfil, 1987; Escobar, 2011; Naz, 2006; Rodríguez, Sarti-Castañeda y Aguilar-Castro, 2015). La desjuvenización (Espinosa, 2015) de las comunidades y territorios rurales e indígenas, favorecida desde las políticas de educación en tono neoliberal e individualista, conjugadas con las políticas que empobrecen el campo y lo niegan como posibilidad de vida, dejan los territorios a expensas de ser apropiados por proyectos extractivos, producción por contrato, monocultivos altamente tecnificados 0 ganadería extensiva; todos estos como veremos más adelante, son procesos descritos por las y los jóvenes del Insittuto Superior Intercultural Ayuuk (ISIA). El despojo y la acumulación por desposesión (Harvey, 2004) que conllevan el etnocidio y la desaparición de los pueblos y sus formas de vida, son confrontados con proyectos educativos interculturales propios (Bertely-Busquets, 2011; Busquets, Dietz, y DíazTepepa, 2013), como el ISIA, gestados desde proyectos de defensa del territorio, de organización política, social y de producción, desde la vida cotidiana, desde acuerdos comunitarios para el cuidado y la conservación de sus territorios y desde disputas en lo legal y jurídico, por el reconocimiento de sus derechos y la autonomía de facto (Bartra, 2013; Bray, 2005; Concheiro-Bórquez y Grajales-Ventura, 2005; Toledo, 1999).

El objetivo de este artículo, es analizar de que forma el ISIA tiene en su origen, en sus planteamientos y en sus procesos político-pedagógicos cotidianos la intención clara de comprometerse con la posibilidad de permanencia de los Pueblos indígenas, especialmente frente a la clara y contundente consigna de su desaparición (SotoSánchez, 2016); esto se recupera al revisar su propuesta de educación intercultural, desde donde teje alianzas por la defensa de sus jóvenes y de los territorios de donde estos provienen, y en particular, nos interesa en este texto, el cómo esto se sintetiza y refleja en el proceso del seminario de titulación (SETI), insertándose con ello en la disputa geopolítica - del saber y de los territorios- que en palabras de las autoridades 
comunitarias es "una lucha no sólo por el territorio, sino por la vida misma" (S. Méndez, comunicación personal, 10 de noviembre 2017).

Este artículo, se realiza en el marco del proyecto de investigación: "Políticas de Interculturalidad en educación superior en Oaxaca: proyectos educativos etnopolíticos", del CONACYT/CIESAS Pacífico Sur. Con una metodología colaborativa, la información que se presenta se obtuvo de documentos del ISIA, notas de periódico, entrevistas en profundidad, encuestas realizadas a los estudiantes, grupos focales con jóvenes y del proceso de facilitación del SETI durante el ciclo 2019-2020 y el actual ciclo 2020-2021 para estudiantes de la carrera de Administración y Desarrollo Sustentable.

\section{La disputa por los territorios y los jóvenes desde los Pueblos Indígenas}

Los territorios indígenas, donde se coproducen la riqueza biocultural y la organización social y política comunitaria (Boege, 2008), enfrentan procesos de despojo y violencia, estamos en riesgo de que en una generación desaparezca una gran parte de la experiencia socio-ecológica profunda que les da origen y sentido (Bartra, 1983; Carton-de Grammont, 2009; Soto-Sánchez, 2016). Los sistemas de conocimiento y sus formas de transmisión, así como la experiencia profunda de los territorios, están siendo abandonados por la adopción de sistemas de producción que degradan y expolian el territorio, el empobrecimiento del campo y la desvalorización de los conocimientos ancestrales de los pueblos.

Todo esto, resultado de procesos complejos y múltiples violencias en donde resalta, para el tema que nos compete, el aumento del reforzamiento ideológico de la escolarización como mecanismo de movilidad social; donde la educación convencional, incompatible con la vida comunitaria no es pertinente frente a las múltiples necesidades, violencias y problemas que enfrentan las comunidades (Saynes-Vásquez, Caballero, Meave, y Chiang, 2013; Soto-Sánchez, 2016); esto último no es sólo mecanismo externo, sino que se reproduce desde el interior de las comunidades, en donde padres de familia y autoridades optan por educación no bilingüe, ni intercultural, reforzando el que las y los jóvenes no puedan permanecer en sus pueblos (Barrón-Pastor, 2008; Corbett, 2000; Maldonado, 2000).

Así, en narrativas que se contradicen, se responsabiliza a las generaciones anteriores - "me mandaron a la escuela, quieren que salga y no vuelva, ya no me enseñaron a hablar la lengua, usan agroquímicos" - o se habla de las nuevas generaciones como los que deciden alejarse y salir - "son indiferentes, no respetan, no quieren trabajar el campo, no les gusta hablar nuestra lengua" (Soto-Sánchez y RoblesGil, 2008; Soto-Sánchez, 2016). En estas narrativas, podemos dar cuenta de la profunda normalización e invisibilización del racismo estructural, las lógicas geopolíticas y de centro-periferia mundiales, resultado de las violencias históricas y actuales hacia los pueblos y sus territorios, mismas que son las causas profundas de 
las problemáticas que se expresan en los pueblos (Acosta et al., 2014; Bartra, 2008; Barrón, 2010; Bonfil, 1987; Escobar, 2011; Naz, 2006, Soto-Sánchez, 2016).

Lo anterior resulta en lo que ya se mencionaba anteriormente, los jóvenes a pesar de no saber qué les espera más allá de sus territorios, buscando abandonar la pobreza y atraso que representan sus pueblos, migran hacia la ciudad de Oaxaca, a México o a los Estados Unidos, que desde el imaginario de la modernidad, se presentan como espacios de posibilidad de una vida mejor (Soto-Sánchez, 2016; Soto-Sánchez y Robles-Gil, 2008; Van Dijk, 2005; Žižek, 2008); esto parece potenciarse frente a la imposibilidad de quedarse, al estar enfrentando, junto con sus comunidades, procesos de deterioro de sus tierras y territorios, espacios limitados 0 nulos para su participación; en los grupos focales, los jóvenes trazan trayectorias de vida que muestran añoranzas del pasado, anhelos de cambiarlo todo y la ilusión de irse para no volver (Grupo focal jóvenes ISIA, comunicación personal, 18 de enero 2019).

Desde la complejidad del escenario descrito arriba, se puede entender la fuerza disruptiva y la posibilidad política de un proyecto educativo planteado desde el referente intercultural y comunitario (González-Apodaca, 2015; Obregón-Alvarez, 2008), cuya intención es que las y los jóvenes aprendan de sus comunidades para refrendar su ontología, epistemologías, sus conocimientos, su historia y las lógicas de vida que de ellos emanan (Blaser, 2009; Chapela, 1999), como semilleros de vida del presente y potencialidades de futuros de vida digna para todos, donde los otros seres que habitan los territorios - plantas, animales, protectores - permanezcan y sobrevivan (Boege, 2008; Escobar et al., 1999; Soto-Sánchez, 2016). Por ello resulta de vital importancia recuperar y analizar la interculturalidad como proyecto etnopolítico (González-Apodaca y Erica, 2009; Walsh, 2009) y la geopolítica que desde el origen dan cimiento y raíz a un proyecto como el Instituto Superior Intercultural Ayuuk (ISIA).

\section{Territorios discontinuos, interconexiones geopolíticas: El origen del ISIA.}

La geografía de origen del ISIA es la región sierra norte, en el estado de Oaxaca, se puede rastrear su raíz hasta el Comité en defensa de los recursos naturales y humanos mixes (CODREMI), quienes al igual que la organización gestada desde pueblos vecinos, la Organización de Defensa de los Recursos Naturales y Desarrollo Social de la Sierra de Juárez (ODRENASIJ), surgieron para la defensa territorial, tomando "la identidad étnica como factor aglutinante" (Chapela, 1999; Bartolomé, 2005: 126), es decir, son movimientos etno-políticos que generaron desde su inicio hasta la fecha procesos educativos tanto formales como informales, construyendo intencionadamente desde lo colectivo y lo comunitario proyectos de transmisión intergeneracional de saberes, es decir de futuro. Estos procesos, hoy en día son raíces de propuestas de Educación Superior; la CODREMI, del ISIA y la ODRENASIJ de la Universidad Autónoma Comunal de Oaxaca (UACO), mismas que 
entretejen personas, procesos y lugares en un horizonte de lucha y defensa de sus jóvenes y sus territorios.

La CODREMI, al paso del tiempo dio lugar a Servicios al Pueblo Mixe (SERMixe), cuyos objetivos han sido el trabajo con la lengua, cultura, autonomía y la defensa de los territorios, siempre vinculados con procesos educativos, mismos que alimentan y nutren el Modelo Educativo del ISIA:

...el ISIA es una iniciativa que tiene como antecedentes el Diplomado en Lengua y Cultura Mixe que se impartió en el año 2000, en la Semana de Vida y Lengua Mixe (SEVILEM) que se lleva a cabo en las comunidades de la región desde hace más de dos décadas, así como en la experiencia que implicó el impulso al modelo del Bachillerato Integral Comunitario (BIC) (...). Todos estos esfuerzos planteaban a la comunidad, el reto de acoger a los egresados del nivel medio superior y atender los procesos de transformación del pueblo Ayuuk hacia el futuro (Modelo Educativo del ISIA, 2014).

En este proyecto, se posiciona desde un ejercicio de derechos, el tener control de la propia educación (Ramírez, 2006; Schmelkes, 2002). Se formula así la creación de "planes, programas, currículas, contenidos y metodologías adecuadas a la realidad y cultura de los pueblos indígenas desde una perspectiva intercultural" (GT Ayuuk, 2004). El Grupo de Trabajo Ayuuk (GT Ayuuk), desde el Centro de Estudios Ayuuk Universidad Indígena Intercultural Ayuuk (CEA-UIIA, A.C.), delineó los principios, formas y fondos que tendría una Universidad Ayuuk Intercultural, a través de la investigación de los sistemas de conocimiento, epistemologías y formas de transmisión del conocimiento en la cultura mixe, buscando: "tener en nuestra propia región una alternativa educativa que no sólo ofrezca calidad, sino que sea pertinente a la cultura Ayuuk, poniendo las condiciones para concretar nuestras aspiraciones de desarrollo integral y sustentable" (GT Ayuuk, 2004).

Por ello, se encuentra dentro del proyecto el corpus político-pedagógico, que desde la noción del "wejën-kajën", se define como:

El desenvolvimiento, descubrimiento y potenciación, continua y permanente de las capacidades, potencialidades, facultades, tendencia y talentos del ser humano-comunero para la creatividad e invención personal y colectiva en tres dimensiones humanas: ja yaa'jkën (nivel biopsíquico) ja wënmää'ny (nivel conocimiento-pensamiento-mente) y ja jää'wën (nivel emocional-espiritual), (Cabildo de Tlahuitoltepec, 2008).

En este caminar, el objetivo que se planteó al CEA-UIIA fue: "identificar, sistematizar, analizar, conocer y difundir los diversos elementos que constituyen la cultura Ayuuk bajo un enfoque intercultural, así como diseñar el modelo educativo de la Universidad Indígena Intercultural Ayuuk (UIIA)" (GT Ayuuk, 2004). 
En la época en que surgía esta iniciativa, en el contexto nacional estaba pujante la creación de las Universidades Interculturales (Dietz, 2014), abanderando un cierto reconocimiento desde el estado a la diversidad cultural; se tuvo un acercamiento para buscar la cobertura del proyecto del CEA-UIIA, pero al final se optó por hacer el acuerdo con el Sistema Universitario de la Compañía de Jesús (SUJ), que en palabras de Ricardo el Ronco Robles: "estaban buscando un proyecto educativo así" (Blanche, 2004), ya que, de acuerdo a David Fernández, tenían intención de buscar a través del enfoque intercultural "evitar y revertir, en la medida de lo posible, el error histórico de haber segregado a los indígenas en su proceso educativo" (Blanche, 2004).

La interculturalidad del ISIA se puede considerar proceso y proyecto, las formas de vivirla, entenderla y soñarla no han sido (ni son) lineales, ni progresivas, ni unívocas. La abstracción del término "interculturalidad" está siempre en disputa, y es relacional, incluso con lo que desde las múltiples alianzas y expectativas se introyecta como "deber ser" (González-Apodaca, 2009; Rojas-Cortés y González-Apodaca, 2016). Así, esta adopta distintas formas y contenidos desde múltiples espacios y por múltiples actores, enmarcado en las relaciones de poder alrededor de las mismas.

Por ello, aunque no es el objetivo de este artículo analizar y discutir esta polivalencia - que puede incluso percibirse como cacofonía de voces- vale la pena delinear que para el caso de este artículo, en el ISIA, la interculturalidad se nombra como tal, en disputa cotidiana con la interculturalidad liberal, desde procesos críticos y espirales de construcción de otras formas de relación entre personas de distintas culturas y pueblos indígenas en y con el espacio escolar de una universidad, pero también con los sistemas de conocimiento y formas sociales de organización de las comunidades de donde provienen las y los jóvenes, y desde el sueño de posibles horizontes de futuro desde sus propios marcos culturales y de relación con sus territorios.

Como ejemplo de lo planteado anteriormente - la polifonía de la interculturalidad- el diseño de las carreras sufrió cambios y adecuaciones, primero en el diálogo con los jesuitas, y posteriormente para lograr los RVOEs, así, aunque aparentemente existe el reconocimiento del Estado de la diversidad cultural, en el terreno de lo concreto, posicionar la realidad y cultura de los pueblos en la educación superior sigue siendo disputado por nociones modernizadoras que plantean que "la mejor alternativa para las comunidades es dejar su cultura, su forma de ser, de pensar y actuar e integrarse a la "cultura y el desarrollo nacional" (GT Ayuuk, 2004).

Por otro lado, el CEA-UIIA finalmente no se estableció en Alotepec, como estaba planeado, lo cual añadió al proceso de creación de la Universidad la búsqueda de un lugar donde poder iniciar este proyecto. La comunidad de Jaltepec de Candayoc, en el municipio de San Juan Cotzocón, se pronunció a favor y se sumó al 
proyecto. Esto generó que la propuesta armada desde SER Mixe y los Jesuitas se entretejiera con un camino comunitario particularmente interesante por el proceso de lucha, defensa territorial, proyectos educativos y de organización de esta comunidad.

Jaltepec es una comunidad mixe, vinculada con los procesos de la región mixe alta, por cuestiones familiares, y también en la defensa de la lengua y cultura y los derechos de los pueblos indígenas, vinculados al trabajo de SER Mixe; además, es un pueblo que ha tenido una participación activa en las luchas históricas por el territorio, que se conjuntaba con un sinfín de procesos que se suscitaron en la región istmo - como la resistencia frente al Plan Puebla Panamá -, acompañada por distintos actores y en especial por la Unión de Comunidades Indígenas de la Zona Norte (UCIZONI) (Guevara-González, 2010; López-Lucio, 2012).

Además, en su preocupación por la escolarización de sus jóvenes, las autoridades de Jaltepec gestionaron un bachillerato comunitario en el año de 1992, el "Bachillerato Asunción Ixtaltepec" (BAI), en acuerdo con otra orden religiosa, los maristas. Este bachillerato estuvo en funciones hasta 2004; las autoridades de Jaltepec gestionaron después otra escuela dentro del sistema oficial y se abrió un Bachillerato Integral Comunitario (BIC).

Por otro lado, como parte de la diócesis de Tehuantepec, reconocida por su alineación a la teología de la liberación, con el obispo Arturo Lona Reyes, en Jaltepec se tenían proyectos, como el bachillerato, las Comunidades Eclesiales de Base, la formación de Catequistas, el acompañamiento e intercambio con organizaciones de productores, como las Comunidades Campesinas en Camino (CCC); con Organizaciones para la defensa de los Derechos Indígenas y del territorio, como el Centro de Derechos Humanos Tepeyac; con procesos de salud comunitaria y tradicional como el Centro Popular de Apoyo y Formación para la Salud (CEPAFOS), una granja de producción porcícola, la producción colectiva de café. Se vinculaban así procesos comunitarios de educación y de defensa del territorio en un tejido del cual el ISIA pasaría a formar parte desde su arranque y que continúa haciéndose presente a través de encuentros, foros y a través del compartir la lucha por los recursos, el territorio y la vida de los pueblos indígenas con organizaciones como las mencionadas aquí, y con otras como Servicios para una Educación Alternativa (EDUCA), los Congresos Comunitarios Sobre Resistencias y Alternativas, el Festival del Agua, entre otras.

\section{Área de influencia del ISIA e implicaciones geopolíticas.}

Al estar esta propuesta vinculada de origen a una diversidad de luchas de los pueblos tanto de la Sierra Norte como del Istmo por sus territorios y por el derecho a una educación que esté en sus manos y para el bien de sus comunidades, este espacio se sigue defendiendo como plataforma desde la cual se disputa la geopolítica del saber y del poder, buscando justicia frente a procesos históricos de discriminación 
y racismo, la disminución de la desigualdad, exclusión y marginación en que se han visto posicionados las y los jóvenes de las comunidades indígenas por las políticas educativas, tanto desde las lógicas de las ofertas que existen, como por su distribución espacial (GT Ayuuk, 2004; Santiago, 2019, comunicación personal; Vázquez, 2017, comunicación personal);

El ISIA atiende principalmente jóvenes de la región mixe y la región istmo del estado de Oaxaca, como se muestra en el Figura 1. Además, han llegado a la institución jóvenes de los estados de Chiapas, Chihuahua, Tabasco y Veracruz. Los jóvenes provienen en su mayoría de comunidades indígenas (mixes, zapotecos, mixtecos, huaves, mazatecos, popolucas, chontales, rarámuri, tseltal, entre otros), donde como ya se ha mencionado, los mecanismos de violencia, despojo y desposesión se articulan de distintas formas, y donde la disputa por los jóvenes y por los territorios está recrudeciéndose, aunque cada región y comunidad con sus particularidades.

\section{Figura 1}

Área de atención del ISIA de acuerdo con el lugar de origen de sus estudiantes.

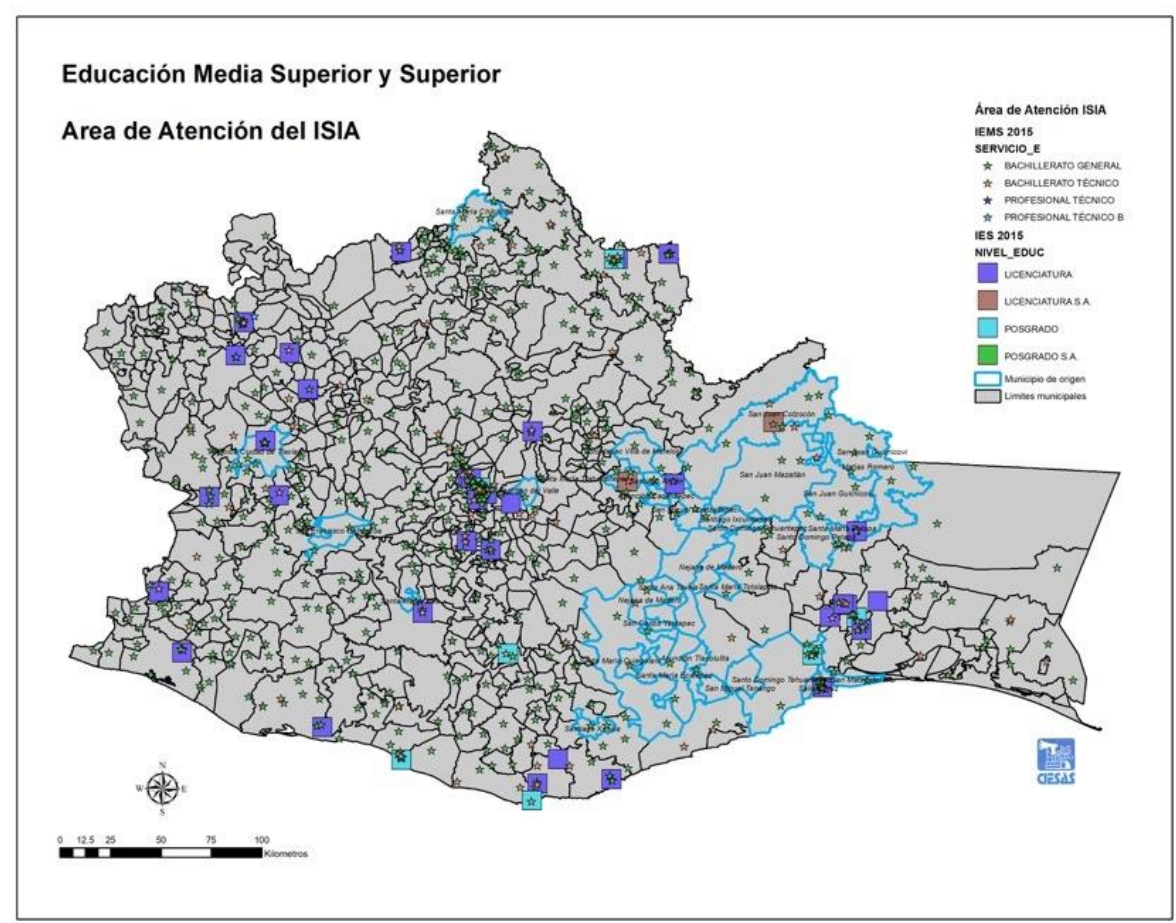

Fuente: Soto-Sánchez, 2019.

A partir del mapa podemos ver que las y los jóvenes que asisten al ISIA provienen principalmente de la región del Istmo de Tehuantepec, que enfrenta al momento conflictos socioambientales por el desarrollo del proyecto del Corredor Transísmico, cuyo origen de acuerdo con lo que plantean algunos estudios data de la época de la Colonia. Este corredor incluye proyectos mineros, de energía, de transporte multimodal, así como corredores industriales, y la producción de 
monocultivos para la exportación (Soto-Sánchez y Robles Gil, 2008; Avila y Avila, 2017; (Luis y Montenegro, 2005; Morosin, 2017)

Por otro lado, en los municipios de donde provienen las y los jóvenes que asisten al ISIA no hay otras instituciones de Educación Superior, esto además de mostrar de algún modo el porqué de la baja cobertura en este nivel educativo en el estado - 19.7\% - refleja una decisión geopolítica de las políticas educativas. Se puede mostrar también que las instituciones de educación superior (IES) se encuentran principalmente en localidades urbanas y cerca del $45 \%$ en la región de Valles Centrales (COEPES, 2010). Destaca que, en el caso del grupo de 18 a 24 años, 84\% de los hombres hablantes de lengua indígena y $88.2 \%$ de las mujeres hablantes no asisten a la escuela (Panorama educativo de la población indígena, 2018). A esto se suma que "al joven indígena no le queda otro camino más que aceptar las carreras existentes, lo cual ha generado un sentimiento de frustración y, a la larga, ha desencadenado el desarraigo cultural y el alejamiento con su comunidad de origen, ya que de regresar simplemente no existirán las condiciones para que pueda desarrollar su profesión" (GT Ayuuk, 2004; Soto-Sánchez, 2016). Es decir, entre las ofertas existentes desde la política educativa del Estado, no se encuentra "una carrera que realmente responda a la compleja realidad de nuestros pueblos" (GT Ayuuk, 2004), sino más bien opciones educativas al servicio de los procesos económicos y de las distintas industrias en los planes de desarrollo.

Así, desde su construcción intercultural (en tensión constante entre la oficial, la liberal y la construida desde abajo) se generan espacios para el diálogo entre distintas personas con distintos saberes, buscando generar condiciones y posibilidades de transformar las realidades de las comunidades desde sus propios referentes y visiones de futuro. Esto enmarcado en otra constante tensión con las lógicas de centro y periferia que conlleva la geopolítica del saber y del poder, los delicados equilibrios entre lo técnico, lo sociocultural y lo político; entre las múltiples y disimiles expectativas y prescripciones dadas por ajenos y propios, aliados y detractores. Con ello, podemos pensar que los procesos etnopolítico- territoriales que configuran el ISIA, dan un carácter específico a su papel en la disputa por la posibilidad de vida y de futuro de los pueblos indígenas más allá de la comunidad donde se encuentra localizado.

\section{Geopolíticas del saber y el poder, construyendo interculturalidad en el SETI}

EI ISIA tiene dentro de las licenciaturas que ofrece, la de Administración y Desarrollo Sustentable, con el objetivo de: "Formar profesionales capaces de diseñar, operar y evaluar proyectos colectivos para la conservación, aprovechamiento, transformación y comercialización de los recursos naturales de la región, desde una perspectiva indígena, intercultural y de la sustentabilidad. En el séptimo y octavo semestre de la Licenciatura, se tiene el Seminario de Titulación (SETI). En diciembre 
de 2019 acordé con la Coordinación Académica del ISIA, acompañar a los estudiantes de esta licenciatura en este seminario.

Había colaborado con otras materias, como Evaluación Rural Participativa y Metodología para el Desarrollo Comunitario, a través de las cuales, a lo largo de los ocho semestres de la licenciatura se van dando elementos y herramientas para que en el séptimo semestre, las y los estudiantes puedan construir un proyecto de investigación comunitaria que de acuerdo al ISIA: "debe de responder a una necesidad sentida y seguir los momentos de la vida comunitaria: la reflexión, la acción y la evaluación" (Santiago, 2019, pp.5).

A partir de este propósito, he facilitado la construcción de proyectos de Investigación Acción Participativa (IAP), en el SETI, en el curso 2019-2020 con 12 estudiantes y en 2020-2021 con 15 estudiantes, todos de distintas localidades. El trabajo ha sido diferenciado en ambos ciclos, por la pandemia de Covid-19, que hizo que en el 2019-2020 se tuviera que suspender el trabajo comunitario, que fue retomado posteriormente y que, en el ciclo actual, significó trabajar el primer semestre con sesiones a distancia, donde se enviaba un video, y posteriormente se iban revisando los trabajos escritos. El proceso de construcción de los proyectos representa encontrar temáticas de disputa geopolítica, para generar procesos de IAP, en los que cada estudiante quiere involucrarse, con su comunidad - o un grupo de ella - para lograr aprendizajes desde una práctica concreta.

Los estudiantes tienen que realizar primero el planteamiento del problema, es decir, cómo está su comunidad, y de qué forma, el tema elegido se ha ido construyendo como problemática. Las narrativas que se van profundizando a lo largo de la construcción de su proyecto de SETI, muestran escenarios complejos y sus miradas a manera de prismas muestran distintas tonalidades de los problemas más comunes en sus comunidades de origen.

Las problemáticas más frecuentes son la expansión de la ganadería, y los monocultivos - como el limón, y el maguey- que casi siempre van acompañados del uso de agroquímicos y de la disminución de la calidad de las tierras y de la producción local para la alimentación. Estos dos problemas - con sus múltiples manifestaciones concretas - generan un fuerte deterioro de las zonas de montaña disminuyendo y afectando los afluentes de agua, situación que repercute en la incertidumbre generalizada de la vida cotidiana. A continuación, algunos extractos de estas problemáticas planteadas por los estudiantes:

Actualmente algunas personas comienzan a talar árboles para sembrar pastos y hacer del terreno un potrero, sin tomar en cuenta los yacimientos (de agua) que existen en ella (Santiago, 2020, pp. 13). 
El uso del suelo para la siembra de maguey espadín (agave angustifolia haw.) ha llevado al límite de reducir poblaciones de especies de animales y plantas que habitan en las zonas deforestadas, como el caso de los magueyes silvestres, venados, iguanas, entre otras. Es notable ver como la cantidad de magueyes silvestres dentro del territorio de la comunidad cada vez es menos; ya que remueven las plantas de agave al momento de rozar los terrenos, evitando así, que se puedan reproducir y servir de alimento para los polinizadores. Además, el ganado bovino representa una fuerte amenaza, ya que les sirven de alimento (Galán, 2020, pp.7).

Una de las grandes preocupaciones de las personas mayores es que, en los últimos 8 años se empezaron a utilizar más el uso de los agroquímicos constantemente en los productos que se cultivan en la comunidad, uno de los temores es que las nuevas generaciones pierdan las costumbres de trabajar en los terrenos como nos enseñaron las generaciones anteriores, como la utilización de yunta, la utilización de abonos de animales, las semillas nativas, etc. (Canseco, 2021, pp. 4).

Un reto importante ha sido el ubicar estas problemáticas como parte de procesos históricos y geopolíticos que amenazan las posibilidades de vida y permanencia de las comunidades en sus territorios. La expansión de la lógica de producción a partir de la explotación de la naturaleza y los seres humanos, es un modelo que se autoreplica y que se reproduce desde las propias personas de la comunidad, esto es, a partir de sus propias familias y de ellos mismos, quienes al buscar mayor ingreso - con mayor eficiencia y desde la disminución del trabajo disponible (Soto-Sánchez y Robles-Gil, 2008); pero como se reflexiona, la violencia a los pueblos es un sistema adaptativo (Žižek, 2008) y esto ha llevado a que este tipo de procesos - de ganadería extensiva y de monocultivos también extensivos - vayan ganando terrenos y por tanto territorios, despojando a la gente de sus conocimientos para producir y haciéndolos cada vez más dependientes del mercado externo y de los insumos químicos.

Hace aproximadamente 25 años el hongo conocido como "roya" afectó drásticamente la producción de café, por ende, el sustento de las familias. A falta de su fuente de ingreso principal, algunas familias se vieron obligadas a adoptar nuevas prácticas de trabajo, ejemplo de esto fue el incremento de la ganadería, sin embargo, no todas las familias tuvieron la misma posibilidad... Con pocas fuentes de ingreso y una necesidad económica creciente, muchas familias de la comunidad optaban por vender sus áreas de trabajo (terrenos de cultivo), quedándose sin un lugar propio en donde producir. Por el contrario, aquellos que si contaban con los recursos financieros suficientes fueron adquiriendo más terrenos, aumentando su capacidad productiva y su oferta de trabajo para los jornaleros de la comunidad.

Fue así como comenzó la normalización del trabajo como "jornaleros", en la ganadería, generando una mayor dependencia económica en la comunidad, 
por su parte, las generaciones más jóvenes optaban por salir de la comunidad a otros lugares en busca de nuevas opciones de educación, así como de ofertas laborales estables en distintos contextos urbanos.

Actualmente las prácticas laborales de Santiago Yaveo y un sistema económico indiferente al sector de producción primario sólo ocasiona que la migración aumente, que las prácticas culturales continúen perdiéndose y que las condiciones de vida sean más favorables para un sector privilegiado de la comunidad, siendo beneficiados exclusivamente aquellos que cuentan con los medios suficientes para solventar los gastos de la ganadería y recientemente la producción de limón, mientras el resto de la población (jornaleros) se beneficia tan sólo del trabajo ofrecido por parte del sectores más empoderados (Lorenzo y Cruz, 2020, pp. 5).

Una vez ubicado el problema, las y los estudiantes se plantean posibles formas de aprender e iniciar el diálogo con sus comunidades; se reconoce que ninguno de los problemas se puede solucionar a partir de un proyecto escolar, sino que son apuestas a mediano y largo plazo, que inician al buscar iniciar la conversación y en algunos casos lograr algunos acuerdos que generen resistencia, frente a la narrativa de desarrollo, que como ideología favorece distintas violencias. De esta forma, el SETI se vuelve una herramienta para soñar junto con otros en sus pueblos posibilidades de futuro para ellos, su agua, sus bosques y sus territorios. Es decir, que generen proceso de desarrollo propio. Como lo presenta Hermelanda en su trabajo:

El trabajo que ahora se presenta al lector o lectora ha sido elaborado con mucho empeño, y representa para mí un doble esfuerzo, porque implicó adentrarse en una metodología que va más allá de una investigación. En donde las personas dejan de ser un objeto de estudio y pasan a ser y formar parte, como compañeros, valorando sus conocimientos y saberes locales, buscando medidas de salvaguardar los bienes comunes y naturales y a solucionar diversas problemáticas vividas en la comunidad (Santiago, 2020, pp.3).

Así, se han ido gestando proyectos donde se ensamblan metodologías participativas para el aprendizaje colectivo, que generan una geopolítica del saber, al poner al centro los conocimientos locales que comúnmente están en manos de viejos y mujeres, para compartirlos con jóvenes y niños, a través de la participación en acciones concretas y de reflexiones comunitarias, que llevan a buscar una geopolítica del poder, es decir que logran retomar el cuidado de los territorios y por tanto inciden en las posibilidades de permanencia de las comunidades y sus jóvenes. En este proceso, las y los jóvenes insertan lo aprendido a lo largo de su formación profesional, su identidad, su pertenencia comunitaria y construyen procesos de interculturalidad que posiblemente les posibiliten poder volver a sus comunidades en el mediano y largo plazo. 
... mi estancia permanente en estos meses (abril-noviembre 2020) de pandemia me permitió darme cuenta que algunas personas ya no quieren cortar los árboles de pino para vender por tres simples razones: 1) esto trae conflictos en el cuidado del ambiente natural, 2) la mala administración económica divide la organización comunitaria, y 3) la desvalorización del pago que dan las empresas madereras; son motivos por los que dicen que es mejor seguir trabajando como se ha hecho en estos 14 años y como lo hacía la gente de antes porque la montaña nos da vida, entonces qué más se necesita cuando ya se tiene lo fundamental para vivir bien. Nuevamente esto me conduce a una reflexión personal. (Antonio, 2020 pp. 106).

El aprendizaje fue mutuo, aunque al inicio no había mucha confianza. Pero, juntos identificamos las posibilidades de organizar y mejorar el uso de los bienes naturales de la comunidad. Por consiguiente, puedo decir que en una investigación de acción participativa necesidades comunitarias. Además de fortalecer y unir la red entre un (profesional-investigador-participantes comunitarios), formando un solo equipo. Así como se encuentran conectados los ríos, mares y océanos.

Asimismo, tener otras miradas y entender que la disminución de los bienes naturales no sólo obedece a la falta de sensibilización de unos cuantos por el territorio o por cierto poderío frente a estos, sino por el contexto en el que se mueven diversos aspectos históricos, culturales, de estructuras de poder, que generan la fractura del tejido social y ambiental en la ciudadanía y que afecta a todo el entorno (Santiago, 2020, pp. 48).

En el ciclo 2019-2020, estas dos estudiantes, Verónica Anotnio y Hermelanda Santiago, lograron que sus proyectos de IAP resultaran en la presentación de las reflexiones obtenidas en el proceso en la asamblea comunitaria, buscando que se aprobaran o generaran propuestas. Así, estas dos mujeres jóvenes presentaron en las asambleas de San Sebastián Jilotepec, de la región Sierra Sur y San Jacinto Yaveloxi, de la región Papaloapan, respectivamente, los procesos de investigación y reflexión sistematizados, que fueron recibidos de manera diferente en las dos comunidades; en el caso de San Sebastián, no hubo acuerdo, aunque los aprendizajes, principalmente de las mujeres y niños, fueron muy significativos; en el caso de San Jacinto se realizó incluso un reglamento comunitario para el cuidado de las tierras comunes y de las fuentes de agua, que pretenden incorporar a los estatutos comunitarios.. Así como estos dos casos, en mayor o menor medida, todos los estudiantes inician procesos de diálogos que recuperan la atención y el interés de otras personas de la población para el cuidado de los territorios.

Otro elemento importante en los SETIs, es que se tiene que presentar en lengua materna - o la lengua indígena que hayan aprendido durante su paso por la universidad - el resumen, y algunas aprendizajes. El tener dentro de los requisitos esto, refuerza la postura del ISIA con respecto a la importancia que tiene las lenguas 
indígenas en los sistemas de conocimiento, epistemologías, y ontologías, y por tanto en la defensa del territorio y el mantenimiento de los sistemas socioecológicos.

\section{Conclusiones}

Las políticas de gobierno, de aumento de la escolarización cuyas ofertas son principalmente orientadas a cumplir con las necesidades de la industria y las empresas extractivas que, sumadas a las políticas de empobrecimiento del campo, y a la discriminación y el racismo hacia las formas de vida de las comunidades campesinas e indígenas, se erigen como sistemas de violencias engarzadas que incrementan la expulsión de jóvenes de las comunidades indígenas y favorecen el despojo de los territorios (Soto-Sánchez, 2016; 2019). Pero la existencia de propuestas educativas, especialmente en el nivel de educación superior, surgidas de procesos etnopolíticos y de defensa del territorio, gestan disputas geopolíticas del saber y el poder; que conllevan la valoración y recuperación de los sistemas de conocimiento propios, es decir interculturales; estos proyectos también enarbolan la búsqueda de reconocimiento, participación y distribución de recursos, como ejercicio de derechos, en la definición de su propia educación y en la construcción de un desarrollo propio desde las culturas y necesidades locales.

Las y los jóvenes que asisten al ISIA provienen de comunidades indígenas que se encuentran principalmente en la región del Istmo, que se ha configurado históricamente desde la disputa geopolítica (López-Lucio, 2012; Martínez-Laguna, Sánchez-Salazar, y Casado Izquierdo, 2002); en esta y otras regiones de Oaxaca y México, se están enfrentando nuevos conflictos a partir de una serie de proyectos extractivos, de desarrollo, e incluso desde proyectos de energía limpia que encierran al final un "capitalismo verde", que resulta igual de expansivo y acaparador (FloresCruz, 2015);

Así, el ISIA, en un camino de constante disputa de la construcción de la interculturalidad, como opción para las comunidades indígenas, buscando permanecer fiel a sus raíces, desde la propuesta del "wejën-kajën"y desde su práctica político-pedagógica, aspira a crear la posibilidad de que las y los jóvenes, en interacción y vinculación con sus comunidades, sueñen y creen proyectos de futuro, delineando así escenarios que continuarán disputando la subalternidad y periferia que se les ha asignado desde la geopolítica mundial del capital.

EI SETI, donde los jóvenes tienen la experiencia de relacionarse desde la IAP con sus comunidades, algunas veces en un retomar la relación que se dejó durante años para estudiar; estas experiencias generan procesos de diálogo y reflexión, donde se conjugan las pertenencias, identidades y conocimientos de la formación profesional, con los conocimientos, preocupaciones y modos de vida de sus propias comunidades, iniciando con ello procesos que esperamos posibiliten no sólo trabajo 
para las y los estudiantes, sino la vida y permanencia de los pueblos y el cuidado de los territorios.

Otro elemento a resaltar del SETI, es la comunidad de aprendizaje generada como grupo de estudiantes del ISIA, en donde se comparten aprendizajes, retos y dificultades del proceso de IAP, esto se transforma en un proceso recursivo de reflexión hacia lo que implica el trabajo en comunidades indígenas, y, sobre todo, las implicaciones de hacer investigación desde dentro, y de intervenir en la comunidad propia. En este mismo tenor, se les ha dado opción a redactar sus trabajos en primera persona, y explicar las implicaciones desde una reflexividad y posicionalidad reflexionada.

Así, antes de convertirse en profesionistas, las y los estudiantes tienen una experiencia de generar desde su creatividad formas de relacionar lo aprendido durante sus estudios con los sistemas de conocimiento locales y sus formas de transmisión, así como la experiencia profunda de los territorios, con distintas generaciones y géneros, desde un lugar diferenciado y respaldado por el ISIA, lo que también les da cierta libertad, y el tiempo para poder reflexionarlo y escribirlo.

Este proceso de interacción con las comunidades de las y los estudiantes, es un espacio que evidencia la interculturalidad que se construye desde el ISIA, y que da una marca específica a las y los jóvenes profesionistas formados ahí, posibilitando sus sueños, que, de acuerdo a sus propias narrativas, incluyen lograr insertarse a los procesos de resistencia existentes o crear junto a sus comunidades nuevos procesos de cuidado y defensa de sus territorios.

\section{Referencias}

Acosta, A., García, A. M., Composto, C., Pérez-Roig, D., Pineda, E., Scheinvar, E., ... Federici, S. (2014). Territorios en Disputa. Despojo capitalista, luchas en defensa de los bienes comunes naturales y alternativas emancipatorias para América Latina. (C. Composito y M. L. Navarro, Eds.) (1era ed.). Ciudad de México: Bajo Tierra Ediciones, Jóvenes en la Resistencia alternativa. www.espora.org/jra

Antonio, V. (2020). Valoración participativa de los servicios ambientales del bosque en la comunidad de san Sebastián Jilotepec [Reporte de Investigación no publicado] Instituto Superior Intercultural Ayuuk.

Avila, A., y Avila Romero, L. E. (2017). Las nuevas Zonas Económicas Especiales en México: despojo agrario y resistencia campesina. The New Special Economic Zones in Mexico: Agrarian Dispossession and Peasant Resistance., $\quad$ 20(40), 138-162. http://search.ebscohost.com/login.aspx?direct=trueydb=a9hyAN=12993572 6ylang=esysite $=$ ehost-live 
Barrón-Pastor, J. C. (2008). ¿Promoviendo relaciones interculturales? Racismo y acción afirmativa en México para indígenas en Educación Superior. Revista Trace, junio(53), 22-35. https://doi.org/10.22134/trace.53.2008.328

Barrón, J. C. (2011). Theorising Intercultural Relations: A Reflection on Cultural Identities, Violence and Emotional Affects in Mexico. Doctoral thesis, School of International Development, University of East Anglia, Reino Unido.

Bartolomé, M.(2005). Visiones de la diversidad I. Relaciones interétnicas e identidades indígenas en el México actual. INAH. México

Bartra-Vergés, A. (2013). Con los pies sobre la tierra "No nos vamos a ir." Alegatos Sección Doctrina, 27(85), 715-750. http://biblioteca.diputados.gob.mx/janium/bv/cesop/lxii/rebciud juscom gu.p df\#page $=388$

Bartra, A. (1983). Las guerras del ogro, 63-106.

Bartra, A. (2008). Campesindios. aproximaciones a los campesinos de un continente colonizado. Boletín de Antropología Americana, (44), 5-24.

Bartra, A. (2013). Con los pies sobre la tierra "No nos vamos a ir." Alegatos Sección Doctrina, 27(85),

715-750. http://biblioteca.diputados.gob.mx/janium/bv/cesop/lxii/rebciud juscom gu.p df\#page $=388$

Bertely-Busquets, M. (2011). Educación superior intercultural en México. Perfiles Educativos, XXXIII(Especial), 66-77. http://www.scielo.org.mx/scielo.php?pid=S018526982011000500007 yscript=sci arttext

Blaser, M. (2009). Political ontology: Cultural studies. Cultural Studies, 23(5), 873896. https://doi.org/10.1080/09502380903208023

Boege, E. (2008). El patrimonio biocultural de los pueblos indígenas en México. Hacia la conservación in situ de la biodiversidad y agrodiversidad en los territorios indígenas (1a ed.). Ciudad de México: Instituto Nacional de Antropología e Historia, Comisión Nacional para el desarrollo de los Pueblos Indígenas.

Bonfil, G. (1987), México profundo, una civilización negada. Grijalbo, México.

Bray, D. (2005). The Community Forests of Mexico, Managing for Sustainable Landscapes. (D. Barton Bray, L. Merino-Pérez, y D. Barry, Eds.) (1st ed.). Austin, TX: University of Texas Press. https://doi.org/10.1659/02764741(2007)27[97:TCFOMM]2.0.CO;2

Busquets, B., Dietz, G., y Díaz-Tepepa, M. G. (2013). Multiculturalismo y educación 2002-2011 (1ERA ed.). Ciudad de México: ANUIES, Consejo Mexicano de Investigación Educativa.

Cabildo de Santa María Tlahuitoltepec, Mixe, Oaxaca, México, (2008). WejënKajën. Las dimensiones del pensamiento y generación del conocimiento comunal, México. 
Canseco, E. (2021). Recuperación de conocimientos tradicionales a través de huertos de traspatio con las familias de la comunidad de Santiago Vargas, San Carlos Yautepec, Oaxaca. [Protocolo de investigación no publicado] Instituto Superior Intercultural Ayuuk.

Carton-de Grammont, H. (2009). La desagrarización del campo mexicano. Convergencia. Revista de Ciencias Sociales, 16(50 mayo-agosto), 13-55. http://www.redalyc.org/articulo.oa?id=10511169002\%0ACómo

CEA-UIIA. (2006). Hacia Dónde Vamos. Un diagnóstico de la región mixe. (C. Cardoso, Ed.). Jaltepec de Candayoc, Oaxaca: Centro de Estudios AyuukUniversidad Indígena Intercultural Ayuuk.

Chapela, F. (1999). Emergencia de las organizaciones sociales de Oaxaca: la lucha por los recursos forestales. Alteridades, 9(17), 105-112. https://alteridades.izt.uam.mx/index.php/Alte/article/view/463

Concheiro-Bórquez, L., y Grajales-Ventura, S. (2005). Movimientos campesinos e indígenas en México: La lucha por la tierra. OSAL: Observatorio Social de América Latina, 16 junio (16), 47-58. http://bibliotecavirtual.clacso.org.ar/ar/libros/osal/osal16/AC16CBorquezGV entura.pdf

Corbett, M. J. (2000). Learning to Leave: The irony of schooling in a coastal community. University of British Columbia, Vancouver, Canada. Retrieved from gc

Dietz, G. (2014). Universidades interculturales.

Escobar, A., Berglund, E., Brosius, P., Cleveland, D. a, Hill, J. D., Hodgson, D. L., Stonich, S. C. (1999). After Nature: Steps to an Antiessentialist Political Ecology. Current Anthropology, 40(1 (Feb.)), pp.1-30. https://www.journals.uchicago.edu/doi/abs/10.1086/515799

Espinosa, G. (2015). La feminización y el envejecimiento resultantes de la migración factores a considerar en las políticas públicas. La jornada del campo. "Agricultura campesina y agroecología". 17 octubre 2015

Flores-Cruz, R. M. (2015). La disputa por el istmo de Tehuantepec: Las comunidades y el capitalismo verde. UAM-XOCHIMILCO.

Galán, S. (2020). Los magueyes silvestres y su importancia para la conservación del medio ambiente en la comunidad de Santa Ana Tavela [Proyecto de Investigación no publicado] Instituto Superior Intercultural Ayuuk.

González-Apodaca, E. (2009). Acerca del multiculturalismo, la educación intercultural y los derechos indígenas en las Américas. Revista de Investigación Educativa 9, julio-dici.

González-Apodaca, E. (2015). Apropaciones escolares en contextos etnopolítocs. Experiencias de egresados de la educación intecultural comunitaria ayuuk. Oaxaca. 
GT Ayuuk (2004). Proyecto de creación de la Universidad Indígena Intercultural Ayuuk. Grupo de Trabajo Ayuuk sobre educación intercultural. Documento de trabajo. Santa María Alotepec Mixe. Oaxaca.

Guevara-González, Y. (2010). Ucizoni y las Organizaciones Sociales en la Zona Mixe Baja Ucizoni y. Diálogo, 13(1), 78-83. Retrieved from http://via.library.depaul.edu/dialogo/vol13/iss1/21

Harvey, D. (2004). El "nuevo" imperialismo: Acumulación por desposesión. Socialist Register, 99-129. https://doi.org/10.1016/0009-2614(71)80574-2

Hernández-Diaz Jorge (2001). "La Asamblea de Autoridades Mixes y Servicios del Pueblo Mixe". en: Reclamos de la identidad: la formación de las organizaciones indígenas en Oaxaca

Lebrato, M. J. (2016). Diversidad epistemológica y praxis indígena en la educación superior intercultural en méxico. Revista Mexicvana de Investigación Educativa, $21(70)$, 785-807. http://www.comie.org.mx/documentos/rmie/v21/n070/pdf/70005.pdf

López-Lucio, C. (2012). La lucha indígena por la dignidad humana. Conflictos socioambientales y derechos humanos en el movimiento indígena del Istmo de Tehuantepec. CIESAS.

Lorenzo, R y Cruz, J. (2021). Diseño de una granja integral en la comunidad de Santiago Yaveo. [Reporte de Investigación no publicado] Instituto Superior Intercultural Ayuuk.

Luis, J., y Montenegro, C. (2005). GEOPOLÍTICA DEL SIGLO XXI EI PPP : Una nueva forma de geopolítica, 1-20.

Maldonado, B. (2000). Los indios en las aulas: Dinamica de dominacion y resistencia en Oaxaca (1era ed.). Oaxaca, Oaxaca, México: Centro INAH Oaxaca.

Martínez-Laguna, N., Sánchez-salazar, M. T., y Casado Izquierdo, J. M. (2002). Istmo de Tehuantepec: un espacio geoestratégico bajo la influencia de intereses nacionales y extranjeros. Éxitos y fracasos en la aplicación de políticas de desarrollo industrial (1820-2002). Boletín Del Instituto de Geografía, UNAM, (49),

118-135. http://www.scielo.org.mx/pdf/igeo/n49/n49a8.pdf

Morosin, A. (2017). Enforcing Accumulation in a Geo-Strategic Region: Paramilitary Threats Against Indigenous Activists in Oaxaca, Mexico. In The Political violencia of capital: paramilitary Formations in Global Perspective (pp. 1-22).

Naz, Farzana (2006). "Arturo Escobar and the Development Discourse: an Overview". Asian Affairs 28(3) 64-84. http://courses.arch.vt.edu/courses/wdunaway/gia5524/Naz06.pdf

Obregón-Alvarez, E. (2008). Wejën Kajë y el Mito de la Educación superior mixe. Ciudad de México. http://congresochiapas08.codigosur.net/ponencias/DFMesadedialogo02.pdf

Ramírez, Elisa (2006). La Educación Indígena en México. UNAM. México. 
Rodríguez, I., Sarti-Castañeda, C., y Aguilar-Castro, V. (2015). Transformación de Conflictos Socio-ambientales e Interculturalidad: Explorando Interconexiones. (A. M. Fernánez, Ed.) (1era ed.). Mérida, Venezuela: Grupo Confluencias, Grupo de Trabajo sobre Asuntos Indígenas del Centro de estudios Políticos y Sociales de América Latina de la Universidad de los Andes de Venezuela, Organización Regional de Pueblos Indígenas de Amazonas.

Rojas-Cortés, A., y González-Apodaca, E. (2016). El carácter interactoral en la educación superior con enfoque intercultural en México. LiminaR. Estudios Sociales y Humanísiticos, 14(1), 73-91. http://www.scielo.org.mx/scielo.php?script=sci arttextypid=S166580272016000100006ylng=esynrm=isoyt|ng=es

Santiago, H. (2020). La participación comunitaria como estrategia de cuidado y defensa del agua en la comunidad de San Jacinto Yaveloxi [Reporte de Investigación no publicado] Instituto Superior Intercultural Ayuuk.

Saynes-Vásquez, A., Caballero, J., Meave, J. A., y Chiang, F. (2013). Cultural change and loss of ethnoecological knowledge among the Isthmus Zapotecs of Mexico. Journal of Ethnobiology and Ethnomedicine, 9(1), 1-10. https://doi.org/10.1186/1746-4269-9-40

Schmelkes, Sylvia (2002). "La enseñanza de la lectura y la escritura en contextos multiculturales". Conferencia presentada en el VII Congreso Latinoamericano para el Desarrollo de la Lectura y la Escritura, Puebla, Pue., 16 al 19 de octubre de 2002. http://red.pucp.edu.pe/ridei/wpcontent/uploads/biblioteca/081212.pdf

SEP. Secretaria de Educación Pública (2015). Reconocimiento de Validez Oficial de Estudios (RVOE). Ingresa al Sistema Reconocimiento de Validez Oficial de Estudios Superiores Federales y Estatales. https://www.gob.mx/sep/acciones-y-programas/reconocimiento-de-validezoficial-de-estudios-rvoe

Soto-Sánchez, A. (2016). Desenmarañando la disputa ontológica: los jóvenes y los territorios entretejidos con las violencias hacia los pueblos indígenas del Istmo de Tehuantepec, Oaxaca. Universidad Autónoma Metropolitana Unidad Xochimilco.

Soto-Sánchez, A. (2019). ¿ Y dónde están los jóvenes ? La Jornada Del Campo.

Soto-Sánchez, A., y Robles-Gil, J. C. (2008). Construcción de la identidad de los jóvenes del istmo de Tehuantepec en sus ámbitos cotidianos de interacción y participación. Universidad Autónoma Metropolitana Unidad Xochimilco. http://www.academia.edu/6607403/UNIVERSIDAD AUTONOMA METRO POLITANA

Toledo, V. M. (1999). El otro zapatismo. Ecología Política, 18, 11-22.

Van Dijk, T. A. (2005). Ideología y análisis del discurso. Utopía y Praxis Latinomericana, $\quad 10(29 \quad$ (Abril-Junio), 9-36. http://www.scielo.org.ve/scielo.php?pid=s131552162005000200002yscript= $\underline{\text { sci arttext }}$ 
Walsh, C. (2009). Interculturalidad crítica y pedagogía de-colonial: apuntes (des)de el in-surgir, re-existir y re-vivir. Educación Intercultural En América Latina. Memorias, Horizontes Históricos y Disyuntivas Políticas., 27-54. https://doi.org/10.1590/S0103-33522013000200004

Žižek, S. (2008). Violence: Six Sideways Reflections (First). New York: PICADOR. Retrieved from www.picadorusa.com 